\title{
REVISION DE LOS CERAMBYCIDAE (COLEOPTERA) DE LA REGION DE MAGALLANES: LISTA ILUSTRADA
}

\author{
REVIEW OF THE LONGHORNS (COLEOPTERA: CERAMBYCIDAE) \\ FROM MAGELLANES REGION: ILLUSTRATED CHECKLIST
}

Álvaro Zúñiga-Reinoso ${ }^{1}$

\section{RESUMEN}

Los Cerambycidae son un grupo de coleópteros bien conocido, carismático y de importancia económica en todo el mundo. En Chile, este grupo no está exento de estas características y ha sido relativamente bien estudiado. Sin embargo, a pesar de la importancia económica, los esfuerzos no han sido suficientes para el conocimiento del grupo y aún persisten problemas taxonómicos, registros dudosos y escaso conocimiento biológico. En particular, no existe una revisión acabada de estos coleópteros en la región de Magallanes. Es por esto que el propósito de este trabajo es compilar, actualizar y entregar nuevos registros de longicornios para la región de Magallanes, complementando el catálogo con ilustraciones, datos sobre su distribución regional y de las plantas hospedadoras. En la literatura se registran siete especies de cerambícidos para Magallanes: Oxypeltus quadrispinosus, Azygocera picturata, Neoholopterus antarcticus, Calydon submetalicum, Microplophorus magellanicum, Callisphyris leptopus y Lautarus concinnus, y se adicionan dos nuevos registros para esta región: Nathrius brevipennis y Chenoderus testaceus. Se discute sobre los registros dudosos, la distribución regional, la asociación con plantas huésped y conservación.

Palabras clave: Longicornios, nuevo registro, especie introducida, distribución regional, plantas hospederas.

\section{ABSTRACT}

The Cerambycidae beetles are a group well-known, charismatic and economic importance in worldwide. In Chile, this group is not exempt from these features and has been a relatively well studied. However, despite the economic importance, efforts have not been sufficient for the knowledge of the group and taxonomic problems persist, uncertain records and little biological knowledge. In particular, there is not detailed review

\footnotetext{
${ }^{1}$ Programa de Doctorado en Ecología y Biología Evolutiva, Facultad de Ciencias, Universidad de Chile, Santiago.
} 
of these beetles in the Magallanes region. That is why the purpose of this paper is to compile, update and give new records of longhorn for the Magellan region, complementing the catalog with illustrations, data on regional distribution and host plants. The literature seven species of longhorn beetles has recorded: Oxypeltus quadrispinosus, Azygocera picturata, Neoholopterus antarcticus, Calydon submetalicum, Microplophorus magellanicum, Callisphyris leptopus y Lautarus concinnus, and two new record is added for this region: Nathrius brevipennis y Chenoderus testaceus. Uncertain records, regional distribution, association with host plants and conservation is discussed.

Key words: longhorn, new record, introduced species, regional distribution, host plants.

\section{INTRODUCCIÓN}

Los Cerambycidae son una familia muy diversa y conocida dentro del orden Coleoptera. Pertenecen a un grupo carismático y apreciado por coleccionistas, debido al gran tamaño de algunas especies, así como también por los vistosos colores metálicos de otras (Peña, 1996). La mayoría de las especies chilenas son difíciles de observar y a la vez biológicamente muy poco conocidas (Peña, 1996). Se les conoce vulgarmente en Chile con el nombre de "longicornios", "taladros" o "sierras", estos dos últimos nombres se debe a que sus larvas son horadadoras de troncos y ramas de plantas leñosas. Es por ello, que muchas especies son de importancia económica dado a que atacan tanto árboles nativos como introducidos de interés forestal y agronómico (Artigas, 1994). Todas estas características, hacen de esta familia un grupo que ha tenido una alta la atención de especialistas en Chile. Los trabajos existentes son en su mayoría trabajos taxonómicos, referentes a la descripción de especies (e.g, Blanchard, 1851; Philippi, 1859; Germain, 1897; Cerda, 1993; Barriga \& Cepeda, 2005; Barriga-Tuñon \& Cepeda, 2007). Existen también el listado sistemático de los Cerambycidae chilenos (Cerda 1986), así como también aparecen mencionados en catálogos de biodiversidad y control de plagas de Chile (e.g Elgueta \& Arriagada, 1989; Artigas, 1994; Peña, 1996; Arias, 2000). Finalmente, existe una variedad de publicaciones que tratan observaciones sobre aspectos biológicos, ecológicos y distribucionales de estos insectos (e.g, Peña, 1960; Fichet \& Barriga, 1990; Barriga et al., 1993; Artigas, 1994; Zúñiga-Reinoso \& Gallegos-Haro, 2010).

En la actualidad, se han descrito alrededor de 8600 especies de cerambícidos para el Neotrópico (Monné, 2005a, Monné, 2005b; Monné, 2006). Sin embargo, solo cerca de 200 especies, entre nativas e introducidas, han sido reportadas para Chile, las que se encuentran distribuidas en seis subfamilias: Paradrinae, Prioninae, Oxypeltinae, Lepturinae, Cerambycinae, Lamiinae (Cerda, 1986; Monné, 2005a, Monné, 2005b, Monné, 2006). Aun así, el número de especies se encuentra en estado de flujo y podría seguir aumentando paulatinamente, ya que aún se siguen encontrando especies nuevas y que han sido recientemente descritas (e.g Barriga \& Cepeda, 2005; Barriga-Tuñon \& Cepeda, 2007).

En la región de Magallanes, a pesar de ser la región con mayor territorio en Chile y con una gran variedad de ecotonos y densos bosques, se han descrito y citado unas pocas especies de cerambícidos (Cerda, 1986). Además, algunas de las especies citadas para Magallanes, tienen registros dudosos y cuyas presencias deben ser confirmadas para la región. A pesar de la baja riqueza, presenta especies de extrema rareza, que recientemente se han podido confirmar y además establecer su hábitat en la región (e.g Zúñiga-Reinoso \& Gallegos-Haro, 2010). La escasez de investigación en la región, así como también el clima y la geografía de esta, son algunos factores que afectan al bajo conocimiento existente sobre los Cerambycidae de la zona. Este trabajo tiene como propósito compilar y actualizar los datos preexistentes de los cerambícidos de Magallanes, entregando nuevos registros y aspectos referentes a la distribución regional, plantas hospederas, especies exóticas y conservación.

\section{MATERIALES Y MÉTODOS}

Se revisó la literatura correspondiente a los Cerambycidae chilenos en busca de antecedentes, claves e ilustraciones, los que fueron usados para la identificación del material de Cerambycidae de Magallanes depositados en diferentes colecciones entomológicas. Las colecciones institucionales, 
colecciones particulares y las muestras entomológicas que fueron revisadas son: Colección de Zoología del Instituto de la Patagonia, Universidad de Magallanes, Punta Arenas, Chile (CZIP); Museo Nacional de Historia Natural, Santiago, Chile (MNHN); Museo de Zoología, Universidad de Concepción, Concepción, Chile (UCCC); Colección de Entomología de la Universidad Metropolitana de Ciencias de la Educación, Santiago, Chile (UMCE); Muestra de Insectos del Parque Nacional Torres del Paine, Torres del Paine, Chile (MITP) y Colección Particular Álvaro Zúñiga, Santiago, Chile (CPAZ).

Por otra parte, se utilizaron sólo las localidades precisas de colecta registradas en colecciones y en la literatura de las especies de cerambícidos de Magallanes. Se trabajó como principal fuente de información bibliográfica y sistemática en base a Monné (2005므, Monné 2006b) y Cerda (1986). Finalmente, se entrega una lámina ilustrada de cada especie para su fácil identificación.

\section{RESULTADOS}

En la bibliografía se registraron 7 especies de longicornios para Magallanes, a la que se le adicionan dos nuevos registros procedente de las colecciones entomológicas revisadas. Los cerambícidos de Magallanes se encuentran distribuidos en cuatro de las seis subfamilias descritas para Chile (Tabla 1). Se detalla a continuación las localidades de colecta regional (cuando corresponda) para cada especie de Cerambycidae registrada en este estudio:

\section{Microplophorus magellanicum Blanchard, 1851}

Distribución en Chile: desde la región del Maule hasta la región de Magallanes y la Antártica Chilena (Cerda, 1986). Localidad de colecta: provincia de Última Esperanza: Parque Nacional Torres del Paine (MITP), Puerto Bellavista (CZIP), Monte Alto (CZIP, MZUC). Provincia de Magallanes: parque Chabunco (CZIP), Reserva Nacional Magallanes (CZIP), Punta Arenas (CZIP, CPAZ, UCCC), jardín botánico del Instituto de la Patagonia (CZIP), Río Seco (UCCC), chorrillo Alicia (UCCC), Puerto del Hambre (CPAZ), río San Juan (CZIP), Tres Puentes (CPAZ, UCCC), Agua Fresca (UCCC). Provincia de Tierra del Fuego: Punta Espora (CZIP). Provincia Antártica Chilena: isla Deceit (CZIP), isla Hoste (CIZP), Puerto Williams (UCCC), isla Navarino (frente isla Snipe) (UCCC).

Callisphyris leptopus Philippi, 1859

Distribución en Chile: desde la región del Maule hasta la región de Magallanes y la Antártica Chilena (Cerda, 1986). Provincia de Última Esperanza:

Tabla 1. Listado de especies y el elenco sistemático de los cerambícidos de la región de Magallanes en base a la revisión bibliográfica y de colecciones entomológicas. * Nuevos registros para la región de Magallanes. += Especie introducida.

\begin{tabular}{|c|c|c|c|c|}
\hline Orden & Familia & Subfamilia & Tribu & Especie \\
\hline \multirow[t]{9}{*}{ Coleoptera } & Cerambicydae & Prioninae & Meroscelicini & Microplophorus magellanicum Blanchard, 1851 \\
\hline & & Lepturinae & Necydalini & Callisphyris leptopus Philippi, 1859 \\
\hline & & Oxypeltinae & Oxypeltini & Oxypeltus quadrispinosus Blanchard, 1851 \\
\hline & & Cerambycinae & Callidiini & Calydon submetalicum (Blanchard, 1851) \\
\hline & & & Biimini & Lautarus concinnus (Philippi, 1859) \\
\hline & & & Holopterini & Neoholopterus antarcticus (Aurivillius, 1910) \\
\hline & & & Unxiini & Chenoderus testaceus (Blanchard, 1851)* \\
\hline & & & Psebiini & Nathrius brevipennis (Mulsant, 1839)+* \\
\hline & & Lamiinae & Acanthoderini & $\begin{array}{l}\text { Azygocera picturata (Fairmaire \& Germain, } \\
\text { 1859) }\end{array}$ \\
\hline
\end{tabular}


Parque Nacional Torres del Paine: campamento Ceron (obs. pers.), camping las Torres (UCCC.). Provincia de Magallanes: Punta Arenas (CZIP, CPAZ, UCCC), jardín botánico del Instituto de la Patagonia (CPAZ), Agua Fresca (UCCC). Provincia de Tierra del Fuego: Punta Espora (CZIP). Provincia Antártica Chilena: parque etnobotánico Omora (obs. pers).

Calydon submetalicum (Blanchard, 1851)

Distribución en Chile: desde la región de Coquimbo hasta la región de Magallanes y la Antártica Chilena (Cerda, 1986). Provincia de Última Esperanza: Parque Nacional Torres del Paine (MITP), Torres del Paine: la península (UCCC), Monumento Natural Cueva del Milodón (UCCC), Monte Alto (CZIP, MZUC). Provincia de Magallanes: parque Chabunco (CZIP), Reserva Nacional Magallanes (CZIP), Punta Arenas (CZIP, CPAZ, UCCC), jardín botánico del Instituto de la Patagonia (CZIP, CPAZ), Río Seco (UCCC), chorrillo Alicia (UCCC), Puerto del Hambre (CPAZ), río San Juan (CZIP), Tres Puentes (CPAZ, UCCC), Agua Fresca (UCCC).

Lautarus concinnus (Philippi, 1859)

Distribución en Chile: desde la región del Maule hasta la región de Magallanes y la Antártica Chilena (Cerda, 1986). Provincia de Última Esperanza: Puerto Natales (CZIP), Monte Alto (UCCC). Provincia de Magallanes: isla Riesco (CZIP), Río Seco (UCCC)

Neoholopterus antarcticus (Aurivillius, 1910)

Distribución en Chile: sólo en la región de Magallanes y la Antártica Chilena (Zúñiga-Reinoso y Gallegos-Haro, 2010). Localidad de colecta: provincia de Última Esperanza: Parque Nacional Torres del Paine (MITP): laguna Amarga (UCCC).

\section{Chenoderus testaceus (Blanchard, 1851)}

Esta especie se distribuye en Chile desde la región del Maule hasta la región de Aysen (Cerda, 1986). Localidad de colecta: provincia de Magallanes: Punta Arenas (UMCE)
Nathrius brevipennis (Mulsant, 1839)

Distribución en Chile: especie de origen europeo e introducida en Chile. En Chile se distribuye desde la región de Tarapacá hasta la región del Maule (Cerda, 1986). Localidad de colecta: provincia de Magallanes: Punta Arenas. (CZIP).

Azygocera picturata (Fairmaire \& Germain, 1859)

Distribución en Chile: desde la región del Biobío hasta la región de Aysén (Cerda, 1986). Sin embargo, Peña (1960) la cita para la región de Magallanes. Provincia Antártica Chilena: faro Yamana, canal Beagle (Peña 1960).

Oxipeltus quadrispinosus Blanchard, 1851

Distribución en Chile: desde la región del Biobío hasta la región de Aysén (Cerda, 1986). Sin embargo, Peña (1976) cita a esta especie como de Magallanes. Provincia de Última Esperanza: Parque Nacional Torres del Paine: lago Dickson (restos de élitros) (obs. pers)

\section{DISCUSIÓN}

De un total de 9 especies de longicornios que se registran en este trabajo para la región de Magallanes, las especies $N$. brevipennis y $C$. testaceus corresponden a un nuevo registro para la región. Esto constituye una ampliación de rango de distribución para ambas especies. Sin embargo, $N$. brevipennis es la primera especie de cerambícido introducida que se registra para la región y la única que se distribuye en todo el país. Al respecto de la introducción de este insecto a Chile poco se conoce, sin embargo ataca a una gran cantidad de plantas introducidas (Barriga et al., 1993), medio por el cual pudo haber sido introducido. Es así que N. brevipennis, debería estar asociado a parques y jardines de Punta Arenas, ya que según Barriga et al. (1993) y Artigas (1994), sus larvas se desarrollan en vegetación leñosa introducida como Salix, Quercus, Pinus, Cupressus y Rosa.

Las especies más comunes de encontrar son $M$. magellanicus, $C$. leptopus y $C$. submetalicum. Siendo M. magellanicus quizás la especie más conocida, 


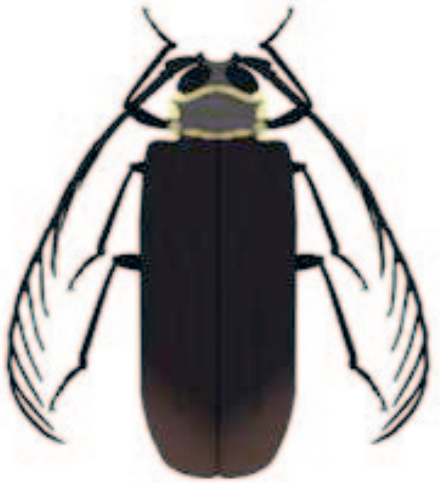

a
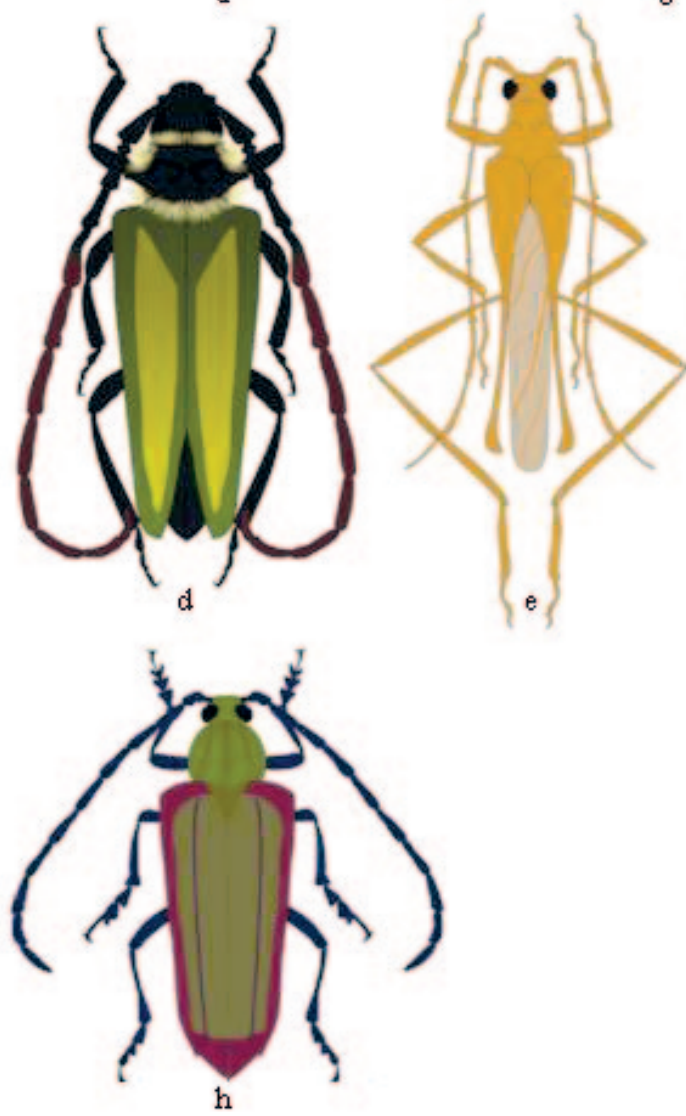

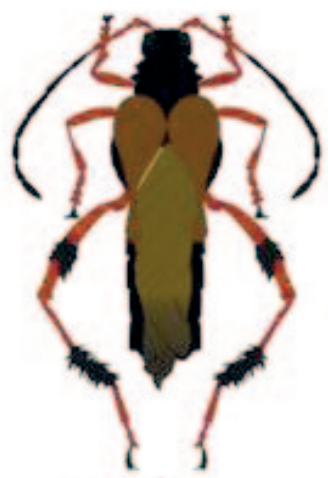

b
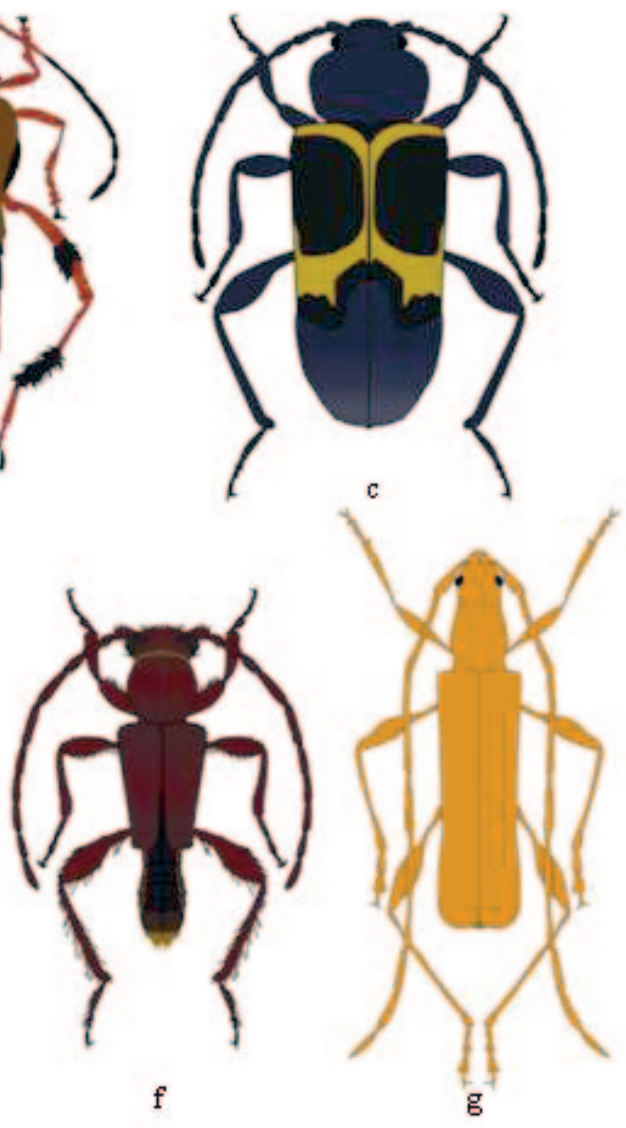

f

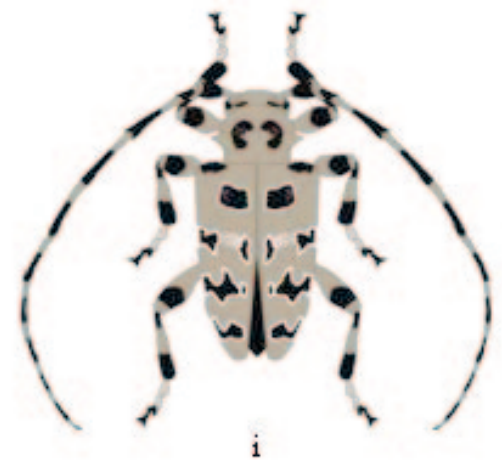

Fig. 1. Cerambícidos de Magallanes: a) Microplophorus magellanicum, b) Callisphyris leptopus, c) Calydon submetalicum, d) Lautarus concinnus, e) Neoholopterus antarcticus, f) Nathrius brevipennis, g) Chenoderus testaceus, h) Oxypeltus quadrispinosus y i) Azygocera picturata. (C) Alvaro Zúñiga-Reinoso. 
debido a que las larvas son comunes de encontrarlas cuando se parte la leña de lenga (Nothofagus pumilio) y es conocida como "gusano blanco de la lenga". Seis de las nueve especies de longicornios que aquí se registran, se encuentran asociadas a los bosques de Nothofagus, ya que las larvas de estas se desarrollan dentro de troncos o ramas de estos árboles. Es por ello, que entre estas especies se encuentran las dos especies que implican mayores daños forestales en la región. Tanto M. magellanicus, como C. submetalicum son barrenadores de troncos de árboles nativos, atacando tres de las cuatro especies de Nothofagus de la región (no se tienen registros de ataque a $N$. nitida), provocando grandes daños en la madera con sus galerías (Artigas, 1995). C. leptopus, también usa estas especies como hospedero, pero atacando las ramas de estos árboles, provocando la caída de estas estructuras (Peña, 1996). Finalmente, nada se sabe sobre la planta hospedera de A. picturata.

La especie $N$. antarcticus, es la que posee la distribución más restringida de la región, encontrándose sólo en el Parque Nacional Torres del Paine (ZúñigaReinoso \& Gallegos-Haro, 2010). Este cerambícido sería la única especie que estaría asociada a vegetación esteparia, que según Zúñiga-Reinoso y Gallegos-Haro (2010), podría estar utilizando como hospedero al arbusto leñoso Mulguraea tridens. Según BarrigaTurron (com. pers.), observó larvas del tipo "sierra" en ramas de $M$. tridens, pero fue imposible criar hasta el desarrollo de los adultos. Por otra parte, probablemente este cerambícido sea una potencial especie amenazada y deba ser considerada en algún estado de conservación. Esto se debe a que existen escasos registros de este insecto desde su descripción (i.e nueve ejemplares desde 1910). Este indicio deja entrever su extrema rareza y su baja densidad poblacional. Por otra parte, son muy raros los cerambícidos que habitan en ambientes tan particulares como la estepa, debido a la escasez de potenciales plantas hospederas leñosas. Finalmente la principal amenaza para este insecto en la actualidad son los reiterados incendios en el Parque Nacional Torres del Paine, particularmente los incendios de los años 2005 y 2011, que han quemado parte de los matorrales de Mulguraea tridens, por lo que se propone poner en alerta el estado de conservación de $N$. antarcticus en la región.

Por otra parte, se debe confirmar la presencia de O. quadrispinosus y A.picturata en Magallanes. Sin embargo, estas especies potencialmente podrían encontrarse en esta región, debido a que ambas poseen registros previos en regiones colindantes, siendo citada ambas para la región de Aysén (Cerda, 1986). Al respecto, el autor halló en el sector del lago Dickson, Parque Nacional Torres del Paine restos de élitros cuya iridiscencia color rojo y puntuación densa hacen pensar que podrían corresponder a $O$. quadrispinosus.

Finalmente, la información aquí entregada sobre los cerambícidos de la región de Magallanes es aún incompleta. Se hace necesario completar la información con más colectas de las especies raras y con pocos registros en la región (i.e N. antarcticus, A. punctatus, O. quadripuntatus, L. concinus y $N$. brevipennis), complementar la información sobre aspectos biológicos de las especies y su asociación con plantas hospederas en la región. Además, entregar más datos sobre la distribución regional. Sin embargo, trabajos recopilatorios como este son necesarios para conocer el estado actual de la diversidad biológica de una región. Además, nos permite conocer cómo se distribuye la biodiversidad en la Patagonia y su asociación con las plantas nativas. También nos permite poner en alerta la llegada de posibles amenazas de especies introducidas y especies con virtuales problemas de conservación. Catálogos de la biodiversidad como este son valiosos al momento de tomar decisiones de protección y conservación para la biodiversidad austral.

\section{AGRADECIMIENTOS:}

El autor agradece sinceramente a Jaime Zavala, por sus aportes literarios y registros de algunas especies. A Vicente Pérez, Jorge Artigas, Patricia Estrada y a Mario Elgueta, por permitir revisar las colecciones del CZIP, UCCC, UMCE y MNHN, respectivamente. A Daniela Mardones por la revisión crítica del manuscrito. Finalmente agradecer a la beca CONICYT № 21110367 para estudios de Doctorados Nacionales.

\section{LITERATURA CITADA}

Arias, E. (2000). Coleópteros de Chile. Santiago, Chile: Fototeknika.

Artigas, J. N. 1994. Entomología Económica. Insectos de interés agrícola y forestal médico y veterinaria. Concepción: Chile: Ediciones Universidad de Concepción. 
Blanchard, E. (1851). Insectos: Coleópteros. In: Gay, C (Ed.), Historia Física y Política de Chile. Zoología (Tomo 5, pp 563). Santiago, Chile. Barriga, J., Curkovic, T., Fichet, T., Henríquez, J. \& Magaya, J. (1993). Nuevos antecedentes de Coleópteros xilófagos y plantas hospederas en Chile, con una recopilación de citas previas. Revista Chilena de Entomología, 20, 65-91.

Barriga, J.E. \& Cepeda, D. (2005). Nuevo género y especie de Compsocerini de Chile (Coleoptera: Cerambycidae). Revista Chilena de Entomología, 30, 5-8.

Barriga, J.E. \& Cepeda, D. (2007). Revisión de los Necydalopsini Blanchard 1851, de Chile y Argentina (Coleoptera: Cerambycidae: Cerambycinae) con descripción de dos nuevos géneros y dos nuevas especies. Revista Chilena de Entomologia, 33, 15-30.

Cerda, M. (1986). Lista sistemática de los cerambícidos chilenos (Coleoptera: Cerambycidae). Revista Chilena de Entomología, 14, 29-39.

Cerda, M. 1993. Nuevos Cerambícidos de Chile (Coleoptera: Cerambycidae). Revista Chilena de Entomología, 20, 13-14

Elgueta, M. \& Arriagada, G. (1989). Estado actual del conocimiento de los coleópteros de Chile (Insecta: Coleoptera). Revista Chilena de Entomología, 17, 5-60.

Fichet, T. \& Barriga, J. E. (1990). Observaciones biológicas sobre Holopterus annulicornis F. Phil., 1859 (Coleóptera: Cerambycidae). Revista Chilena de Entomología, 18, 61-65.

Germain, P. (1897). Apuntes entomolójicos. Los lonjicornios chilenos. Anales de la Universidad de Chile, 97, 423-459.

Monné M.A. (2005a). Catalogue of the Cerambycidae (Coleoptera) of the Neotropical Region. Part I. Subfamily Cerambycinae. Zootaxa, 946, 1-765.

Monné M.A. (2005b). Catalogue of the Cerambycidae (Coleoptera) of the Neotropical Región. Part II. Subfamily Lamiinae. Zootaxa, 1023, 1-759.

Monné M.A. (2006). Catalogue of the Cerambycidae (Coleoptera) of the Neotropical Región. Part III. Subfamilies Parandrinae, Prioninae, Anoplodermatinae, Aseminae, Oxypeltinae, Spondylidinae, Lepturinae and addenda to the Cerambycinae and Lamiinae. Zootaxa, 1212, 1-244.

Peña, L. (1960). Algunas observaciones sobre especies poco conocidas de Cerambycidae (InsectaColeoptera) de Chile. Revista Universitaria Católica de Chile, 44-45, 57-61.

Peña, L. (1976). Guía para reconocer los coleopteros de Chile continental. Expedición a Chile. Santiago, Chile: Editorial Nacional Gabriela Mistral.

Peña, L. (1996). Introducción al estudio de los insectos de Chile. Santiago, Chile: Editorial Universitaria.

Philippi, F. (1859). Algunas especies nuevas de coleópteros de la provincia de Valdivia. Anales de la Universidad de Chile, 16, 656-678.

Zúñiga-Reinoso A. \& Gallegos-Haro, G. (2010). Registros y antecedentes sobre el rango de distribución de Neoholopterus 
Á. ZÚÑIGA-REINOSO 